\title{
iGEMDOCK: a graphical environment of enhancing GEMDOCK using pharmacological interactions and post-screening analysis
}

\author{
Kai-Cheng Hsu ${ }^{1 \dagger}$, Yen-Fu Chen ${ }^{1 \dagger}$, Shen-Rong Lin ${ }^{1}$, Jinn-Moon Yang ${ }^{1,2,3^{*}}$ \\ From The Ninth Asia Pacific Bioinformatics Conference (APBC 2011) \\ Inchon, Korea. 11-14 January 2011
}

\begin{abstract}
Background: Pharmacological interactions are useful for understanding ligand binding mechanisms of a therapeutic target. These interactions are often inferred from a set of active compounds that were acquired experimentally. Moreover, most docking programs loosely coupled the stages (binding-site and ligand preparations, virtual screening, and post-screening analysis) of structure-based virtual screening (VS). An integrated VS environment, which provides the friendly interface to seamlessly combine these VS stages and to identify the pharmacological interactions directly from screening compounds, is valuable for drug discovery.

Results: We developed an easy-to-use graphic environment, iGEMDOCK, integrating VS stages (from preparations to post-screening analysis). For post-screening analysis, iGEMDOCK provides biological insights by deriving the pharmacological interactions from screening compounds without relying on the experimental data of active compounds. The pharmacological interactions represent conserved interacting residues, which often form binding pockets with specific physico-chemical properties, to play the essential functions of a target protein. Our experimental results show that the pharmacological interactions derived by iGEMDOCK are often hot spots involving in the biological functions. In addition, iGEMDOCK provides the visualizations of the protein-compound interaction profiles and the hierarchical clustering dendrogram of the compounds for post-screening analysis.
\end{abstract}

Conclusions: We have developed iGEMDOCK to facilitate steps from preparations of target proteins and ligand libraries toward post-screening analysis. iGEMDOCK is especially useful for post-screening analysis and inferring pharmacological interactions from screening compounds. We believe that iGEMDOCK is useful for understanding the ligand binding mechanisms and discovering lead compounds. iGEMDOCK is available at http://gemdock.life.nctu.edu.tw/dock/ igemdock.php.

\section{Background}

Structure-based drug design is widely used to identify lead compounds with the growing availability of protein structures [1-3]. Many tools (e.g., GEMDOCK [4], DOCK [5], AutoDock [6], and GOLD [7]) have been developed for virtual screening (VS) and successfully identified lead compounds for some target proteins.

\footnotetext{
* Correspondence: moon@faculty.nctu.edu.tw

+ Contributed equally

${ }^{1}$ Institute of Bioinformatics and Systems Biology, National Chiao Tung University, Hsinchu, 30050, Taiwan

Full list of author information is available at the end of the article
}

However, the accuracy of these docking tools remained intensive because of the incomplete understandings of ligand binding mechanisms [1-3]. In addition, most of scoring functions are lack of pharmacological interactions that are essential for ligand binding or biological functions [8]. Recently, some approaches have been proposed to derive pharmacological interactions from known compounds [8-10]. These approaches apparently increase hit rates for identifying the active compounds which are often similar to the known compounds. In addition, these approaches are often unable to be 
applied for new targets, which have no known active compounds.

Generally, a VS procedure consists of four main steps: preparations of the target protein and the compound library, docking and post-screening analysis (e.g., clustering compounds and pharmacological interactions). Most docking programs (e.g. DOCK [5] and AutoDock [6]) only provide docked poses or loosely coupled these steps. They often provided limit ability for post-screening analysis. Therefore, a VS framework, providing an easy-to-use graphic and integrated environment, is an emergent task for drug discovery.

To address these issues, we have developed a structure-based VS framework, named $i$ GEMDOCK, from preparations through to post-screening analysis. $i$ GEMDOCK is an integrated environment, which integrates the heavily modified and enhanced in-house tool GEMDOCK, protein-ligand profiles, pharmacological interactions, and compound clusters. GEMDOCK was comparative to several docking tools (e.g. DOCK [5] and GOLD [7]) and has been successfully applied to identify new inhibitors and new binding sites for some targets [4,8,11-14]. Notably, iGEMDOCK derives the pharmacological interactions from screening compounds without using a set of known active compounds. The pharmacological interactions, which often form binding pockets with specific physico-chemical properties of the target protein, can represent conserved interactions between the interacting residues and the screening compounds. We initially validated the pharmacological interactions on three therapeutic protein targets, including estrogen receptor $\alpha$ for antagonists and agonists and thymidine kinase. Our experimental results show that the derived pharmacological interactions are often essential for the ligand binding or maintaining biological functions for these targets. In addition, $i$ GEMDOCK provided a postscreening analysis module, which is convenient for clustering compounds and visualizing the pharmacological interactions by interaction profiles. We believe that $i$ GEMDOCK is useful for drug discovery and identifying essential residues and interactions for understanding the binding mechanisms.

\section{Methods}

\section{Preparations of proteins and compound sets}

To initially validate the pharmacological interactions, we selected three therapeutic protein targets, including estrogen receptor $\alpha$ for agonists (ERA, PDB code 1gwr [15]), estrogen receptor $\alpha$ for antagonists (ER, PDB code 3ert [16]), and thymidine kinase (TK, PDB code 1 kim [17]) because these proteins were well studied. The catalytic mechanisms, biological functions, key functional residues, and active compounds of the three targets were available in the literatures. Estrogen receptor is an important therapeutic target for osteoporosis and breast cancer [18], and TK is a drug target for the therapy of herpes simplex virus type-1 [19]. Moreover, we also evaluate the docking and screening accuracy of $i$ GEMDOCK. For docking, a highly diverse dataset comprising 305 protein-compound complexes (i.e., CCDC/Astex set [20]) was selected; for screening, we prepared 10 known active compounds and 990 compounds were randomly selected from Available Chemical Directory (ACD) proposed by Bissantz et al. [21] for each therapeutic protein target.

\section{Main procedure}

$i$ GEMDOCK is an integrated VS environment from preparations through post-screening analysis with pharmacological interactions (Fig. 1A). First, iGEMDOCK provides interactive interfaces to prepare both the binding site of the target protein and the screening compound library (Figs. 1B and 1C). Each compound in the library is then docked into the binding site by using the in-house docking tool GEMDOCK [4]. Subsequently, $i$ GEMDOCK generates protein-compound interaction profiles of electrostatic $(\mathrm{E})$, hydrogen-bonding $(\mathrm{H})$, and van der Waals $(\mathrm{V})$ interactions. Based on these profiles and compound structures, iGEMDOCK infers the pharmacological interactions and clusters the screening compounds for the post-screening analysis (Figs. 1D and $1 \mathrm{E})$. Finally, $i$ GEMDOCK ranks and visualizes the screening compounds by combining the pharmacological interactions and energy-based scoring function of GEMDOCK.

\section{Mining pharmacological interactions}

$i$ GEMDOCK mines the pharmacological interactions based on protein-compound interaction profiles (Fig. 2). The size of each profile is $N \times 2 K$ where $N$ and $K$ are the numbers of screening compounds and interacting residues of the target protein, respectively. Here, an interacting residue is divided into two interacting groups: main and side chains. A profile $P(I)$ with type $I(\mathrm{E}, \mathrm{H}$, or $\mathrm{V})$ is given as (Fig. 2A):

$$
P(I)=\left[\begin{array}{cccc}
p_{1,1} & p_{1,2} & \cdots & p_{1,2 K} \\
p_{2,1} & p_{2,2} & \cdots & p_{2,2 K} \\
\vdots & \vdots & \ddots & \vdots \\
p_{N, 1} & p_{N, 2} & \cdots & p_{N, 2 K}
\end{array}\right]
$$

where $p_{i, j}$ is a binary value ( 0 or 1 ) for the compound $i$ interacting to the residue group $j$. In the $\mathrm{E}$ and $\mathrm{H}$ profiles, the $p_{i, j}$ is set to 1 (green) if hydrogen-bonding or electrostatic interactions are yielded between the compound $i$ and the residue $j$ (energy $\leq-2.5 \mathrm{kcal} / \mathrm{mol}$ ); otherwise, $p_{i, j}=0$ (black). For the V profile, $p_{i, j}=1$ if the interacting energy is less than $-4 \mathrm{kcal} / \mathrm{mol}$ (Fig. 2A). 


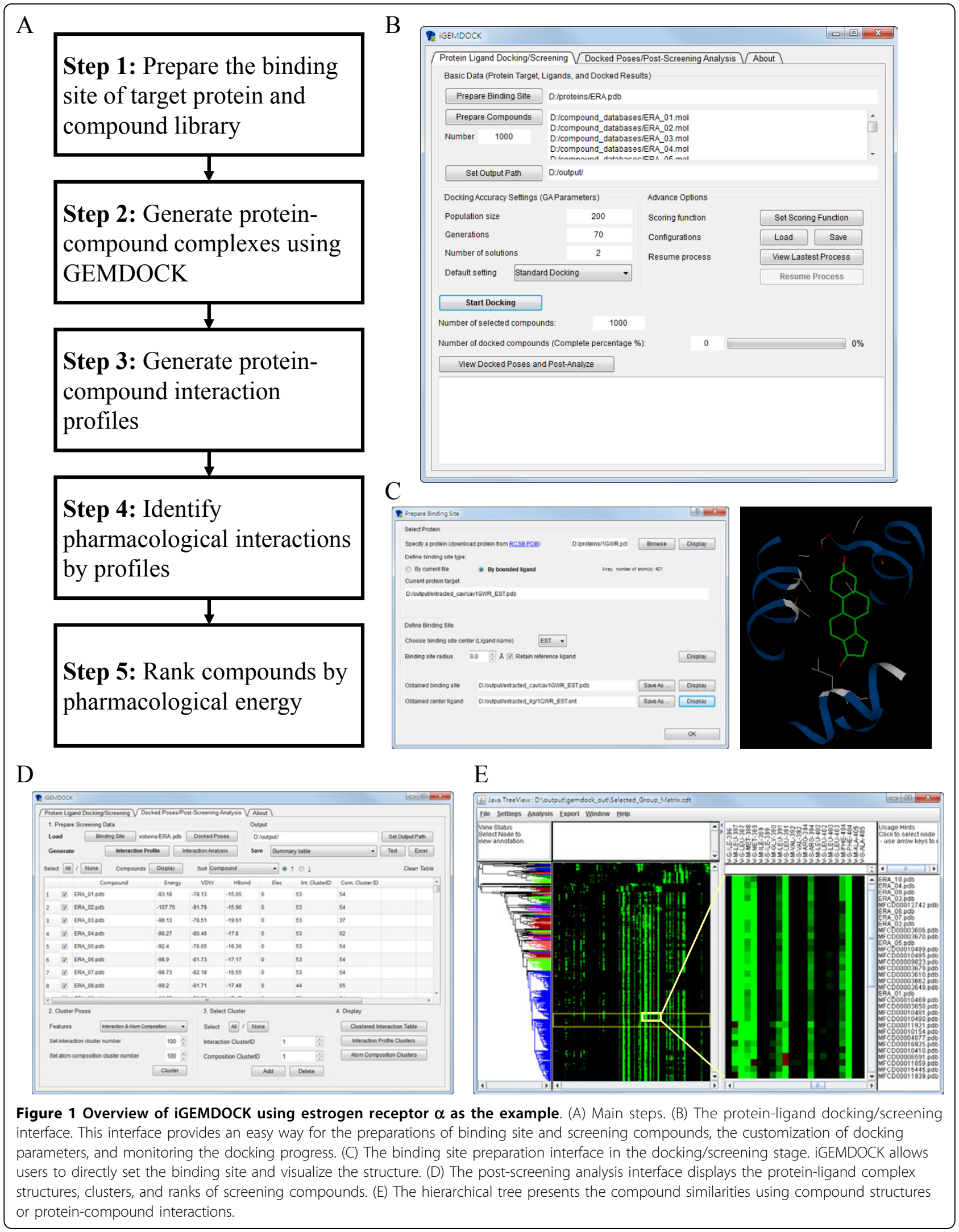




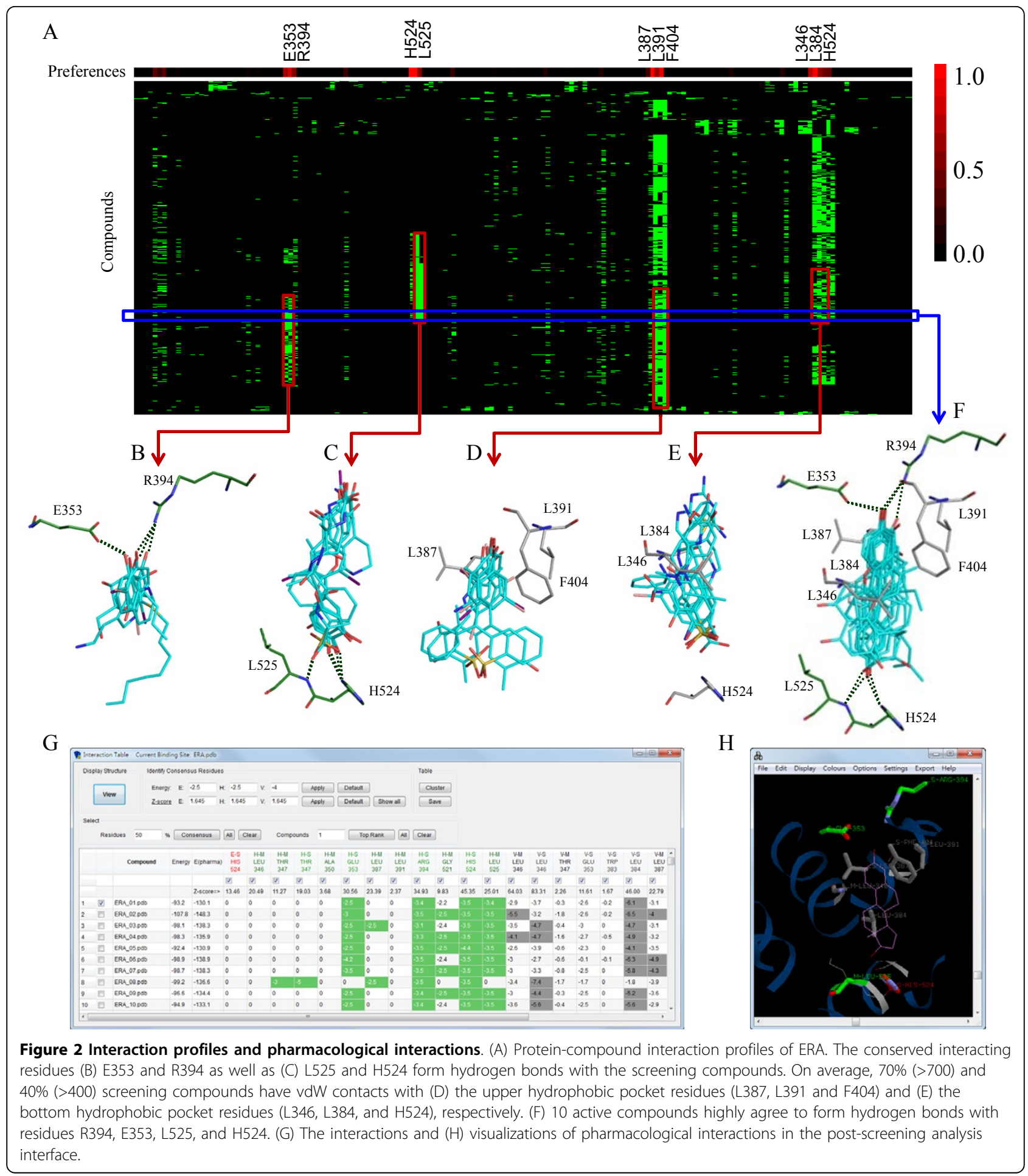

After the generations of the profiles, we identified the pharmacological interactions. For each interacting residue group, the $z$-score value is used to measure the interaction conservation between the interacting groups and the screening compounds. For computing the zscores of interacting groups in a profile, 1000 randomly shuffled profiles are utilized to obtain the standard deviation $(\sigma)$ and mean $(\mu)$. The $z$-score of the interacting residue group $j$ is defined as $Z_{j}=\frac{f_{j}-\mu}{\sigma}$, where $f_{j}$ is given as $f_{j}=\sum_{i=1}^{N} \frac{p_{i j}}{N}$, where $N$ is the number of 
screening compounds. Finally, we normalize the $z$-score value as follows:

$$
W_{j}=\left\{\begin{array}{cc}
z_{j} / z_{\max } & \text { if } z_{j}>0 \\
0 & \text { if } z_{j} \leq 0
\end{array}\right.
$$

where $W_{j}$ is the interaction conservation of the residue group $j$ related to the largest $\mathrm{z}$-score $\left(z_{\max }\right)$ among all of interacting groups in the binding site. Here, an interaction conservation is viewed as a pharmacological preference and an interaction is considered as the pharmacological interaction if $W_{j} \geq 0.4$. For example, for the hydrogen profile of the target ERA, the pharmacological preferences of E353 and R394 are 0.64 and 0.80, respectively; for the V profile, the preferences of L387, L391, and F404 are 1.00, 0.61 , and 0.90 , respectively (Fig. 2B). In this case, over 300 $(>30 \%)$ screening compounds form hydrogen bonds with the residues E353 or R394 by polar moieties (e.g., hydroxyl group (27\%), carboxyl group (20\%), sulfuric acid monoester $(9 \%)$, ketone $(8 \%)$, and phosphoric acid monoester (6\%)). Moreover, the aromatic rings of the screening compounds are often sandwiched by vdW interacting residues L387, L391, and F404 (Fig. 2D).

Based on the pharmacological interactions, we developed a pharmacological scoring function for identifying the active compounds from thousands of screening compounds. The pharmacological scoring function is given as

$$
E_{\text {pharma }}=E_{G E M D O C K}+E(\mathrm{E})_{\text {pharma }}+2 E(\mathrm{H})_{\text {pharma }}+0.5 E(\mathrm{~V})_{\text {pharma }}(2)
$$

where $E_{G E M D O C K}$ is the docked energy of GEMDOCK and $E(\mathrm{E})_{\text {pharma }}, E(\mathrm{H})_{\text {pharma }}$, and $E(\mathrm{~V})_{\text {pharma }}$ are the pharmacological scores of electrostatics, hydrogen-bonding, and $\mathrm{vdW}$ interactions, respectively. The $E(I)_{\text {pharma }}$ with interaction type $I$ (i.e., E, H, or V) is defined as

$$
E(I)_{\text {pharma }}=\sum_{j=1}^{2 K} W_{j} e_{j}
$$

where $e_{j}$ is the energy obtained by the GEMDOCK scoring function for the residue group $j$. Finally, $i$ GEMDOCK provides the ranks of energy-based and pharmacological scoring functions for all screening compounds.

\section{Implementation of iGEMDOCK}

$i$ GEMDOCK is an easy-to-use VS environment and includes three main modules (Fig. 1): docking and virtual screening tool (GEMDOCK); post-screening analysis methods; and visualization tools (RasMol [22] and Java Treeview [23]). We employed ERA as an example to present these modules, procedures and features of $i$ GEMDOCK.
For protein-ligand docking/screening module, iGEMDOCK provides an interactive interface for the preparations of the binding site and compound library; setting docking parameters; and monitoring progress status (Fig. 1B). For most docking tools, users usually need to prepare the binding site structure and compound library through complicated steps (e.g., add hydrogen atoms and generate the grids of the protein). Here, iGEMDOCK provides a straightforward method to derive the binding site from the bounded ligand. For example, the binding site of ERA (PDB code 1gwr) was obtained from the estradiol (Fig. 1C), $i$ GEMDOCK is able to automatically consider the effects of hydrogen atoms when preparing the binding site and the compound library. In addition, $i$ GEMDOCK allows users to visualize and refine the binding site of the target protein. Additionally, iGEMDOCK offers the similar way to prepare screening compounds and docking parameters (e.g., the population size and the number of generations).

After the screening process, $i$ GEMDOCK utilizes the post-screening analysis module to infer pharmacological interactions and cluster screening compounds based on protein-ligand complexes and compound structures (Fig. 1D). First, $i$ GEMDOCK generates interaction profiles and calculates the pharmacological preference $\left(W_{j}\right)$ of each interacting group for deriving the pharmacological interactions (Fig. 2). These pharmacological preferences and interactions are shown in an interactive window (Fig. 2G); furthermore, RasMol displays the pharmacological interactions with conserved interacting residues and functional groups of compounds (Fig. 2H). Additionally, iGEMDOCK supports a hierarchical clustering method to cluster screening compounds according to interaction profiles and the atomic composition (Fig. 1E). The atomic composition, which is similar to the amino acid composition of a protein sequence, is useful for measuring compound similarity. $i$ GEMDOCK provides an interactive interface for visualizing compound similarity with a hierarchical tree by Java Treeview. Finally, $i$ GEMDOCK ranks and visualizes the screening compounds by combining the pharmacological interactions and the energy-based scoring function.

\section{Results and discussion}

Pharmacological interactions

The pharmacological interactions derived by $i$ GEMDOCK are often involved in biological reactions or essential for ligand binding. We examined the pharmacological interactions on three selected target proteins (ERA, ER, and TK). First, we compared the pharmacological interactions, derived from 1000 screening compounds, to the consensus interactions, derived from 10 active compounds (Table 1 and Fig. 3). Here, the residue 
Table 1 Pharmacological interactions and consensus interaction ratio on estrogen receptor $\alpha$ and thymidine kinase

\begin{tabular}{|c|c|c|c|}
\hline Protein & $\begin{array}{c}\text { Predicted pharmacological } \\
\text { interactions }\end{array}$ & $\begin{array}{c}\text { Consensus interaction } \\
\text { ratio }^{\text {a }}\end{array}$ & Related works \\
\hline \multirow[t]{10}{*}{ ERA } & $\mathrm{R} 394-\mathrm{H}^{\mathrm{b}}\left(0.80^{\mathrm{c}}\right)$ & 1.0 & Form hydrogen bonding networks for ligand binding $[26,27]$ \\
\hline & E353-H (0.64) & 0.8 & \\
\hline & $\mathrm{H} 524-\mathrm{H}(1.00)$ & 1.0 & \\
\hline & L387-V (1.00) & 0.9 & Form non-polar contacts with A-ring of sterols scaffolds $[28,29]$. \\
\hline & L387-H (0.52) & 0.2 & \\
\hline & F404-V (0.90) & 1.0 & \\
\hline & V346-V (0.98) & 0.5 & \\
\hline & L391-V (0.61) & 0.9 & \\
\hline & L384-V (0.57) & 0.9 & \\
\hline & L525-H (0.53) & 0.9 & \\
\hline \multirow[t]{14}{*}{ TK } & R222-H (1.00) & 0.8 & Transfer phosphate in the substrate phosphorylation $[30,31,36]$ \\
\hline & R222-E (1.00) & 0.0 & \\
\hline & R222-V (0.62) & 0.4 & \\
\hline & R163-H (0.99) & 0.6 & \\
\hline & R163-E (0.40) & 0.0 & \\
\hline & R163-V (0.56) & 0.8 & \\
\hline & E83-V $(0.54)$ & 0.2 & \\
\hline & Y101-H (0.40) & 0.2 & $\begin{array}{l}\text { Form hydrogen bonds with thymidine; activity was decreased over } 90 \% \text { if } \\
\qquad \text { Q125 mutated [32] }\end{array}$ \\
\hline & Y101-V (0.45) & 0.0 & \\
\hline & Q125-H (0.40) & 1.0 & \\
\hline & Y172-V (1.00) & 1.0 & Sandwich the thymine moiety of substrates [33] \\
\hline & M128-V (0.58) & 1.0 & \\
\hline & W88-V (0.87) & 0.9 & Constitute a pocket for ligand binding [33] \\
\hline & H58-V (0.68) & 0.9 & \\
\hline
\end{tabular}

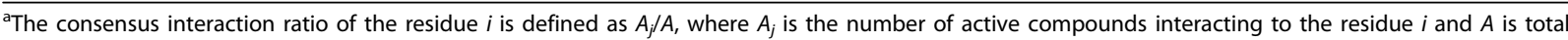
number of active compounds.

${ }^{\mathrm{b}} \mathrm{H}, \mathrm{E}$ and $\mathrm{V}$ are the interaction types.

'The pharmacological preferences (i.e. $W_{j}$ defined in Equation (1)).
}

$i$ is considered as "hot spot" if the consensus interaction ratio $\geq 0.5[9,10,24,25]$. Among 10 predicted pharmacological interactions (residues) for ERA, 9 pharmacological interactions ( 9 of 9 residues) agree with hot spots except the L387 with the hydrogen-bonding interaction. For TK, 8 of 14 pharmacological interactions (7 of 9 residues) are the hot spots. These results indicate the pharmacological interactions (residues) from screening compounds are often essential for the ligand binding. For example, 10 active compounds of TK form stacking interactions with the residue $\mathrm{Y} 172$ (vdW preference is 1.0 defined in Equation (1)) that stabilizes the binding of thymine or purine moieties.

We also examined the pharmacological interactions by their biological functions or binding mechanisms. For estrogen receptor $\alpha$, H524 (hydrogen-bonding preferences are 1.0 and 0.42 for ERA and ER, respectively) is involved in a hydrogen-bonding network [26]; similarly, E353 and R394 (hydrogen-bonding preferences $\geq 0.5$ for both ERA and ER) interact the structural water to form the hydrogen bonding network (Table 1 and Fig. 3) [27].
These two hydrogen bonding networks are essential for estrogen receptor modulators to trigger the responses of estrogen receptor $\alpha[26,27]$. For ER and ERA, hydrophobic interacting residues, L346, L387, F404, and L525 with high vdW interaction preferences, contact with the sterols or flavones scaffolds of the active compounds. These residues contribute the major vdW interactions for the ligand binding of estrogen receptor $\alpha[28,29]$.

For TK, R222 and R163 play major roles for inhibitor and substrate binding [30,31], and their hydrogen-bonding preferences are 1.0 and 0.99 , respectively (Table 1 ). Our method identified the electrostatic interactions of R222 and R163 (preferences are 1.0 and 0.4, respectively), which help to transfer phosphate in the substrate phosphorylation [30]. However, these two electrostatic interactions are not observed from 10 active compounds (Fig. 3). For the residue Q125 (H preference 0.40), the TK activity was decreased over 90\% if Q125 mutated into Asp, Glu, or Asn [32]. The residues M128, Y172, $\mathrm{H} 58, \mathrm{R} 163$, and Y88 constitute a pocket to fix the substrate, and their vdW preferences are $0.58,1.00,0.68$, 
A

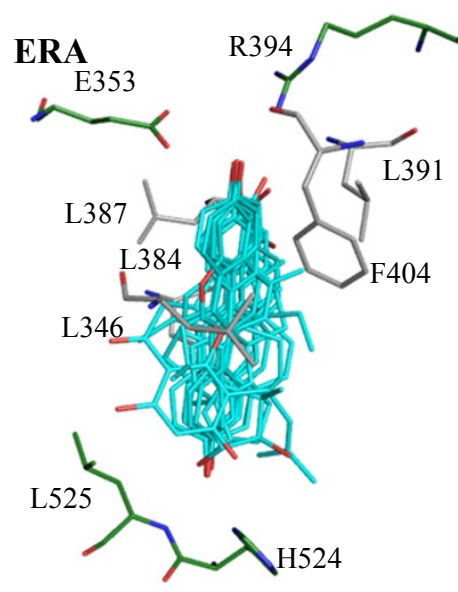

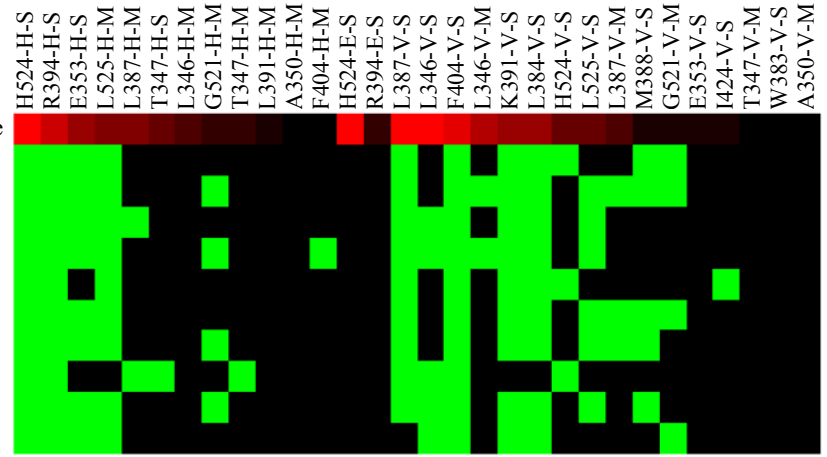

B
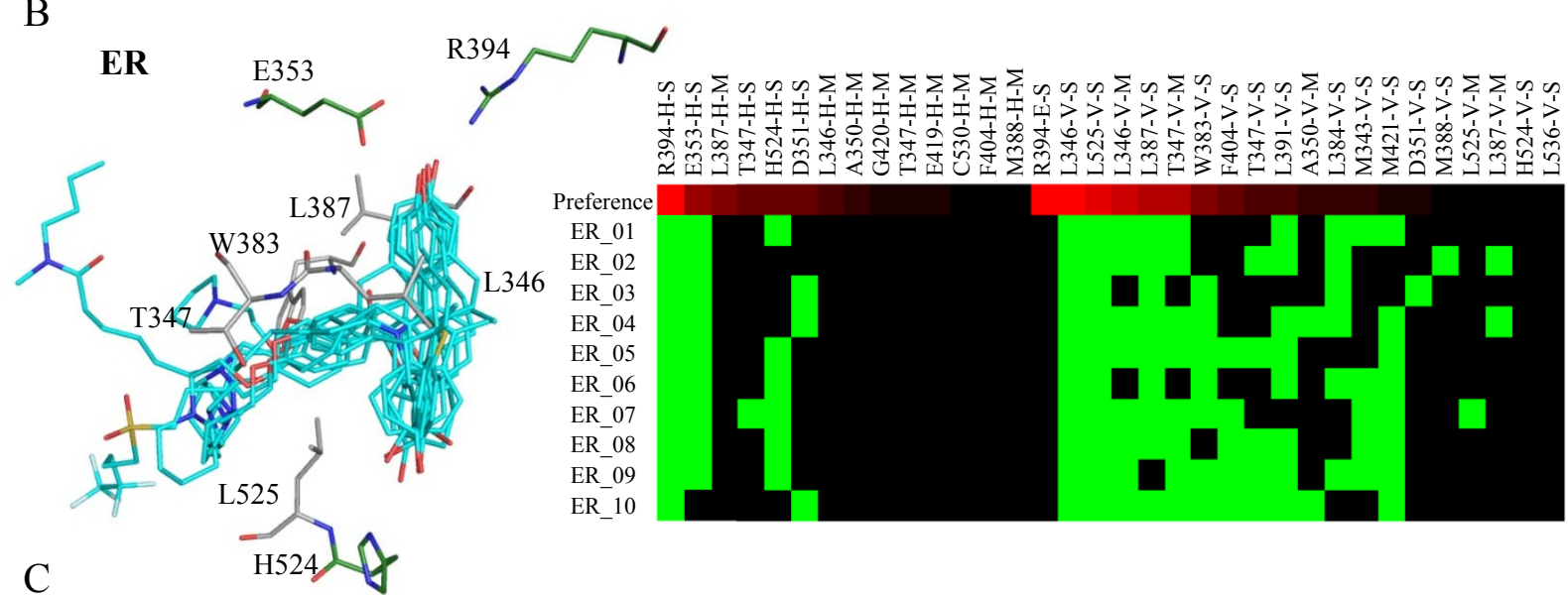

$\mathrm{C}$

\section{TK}
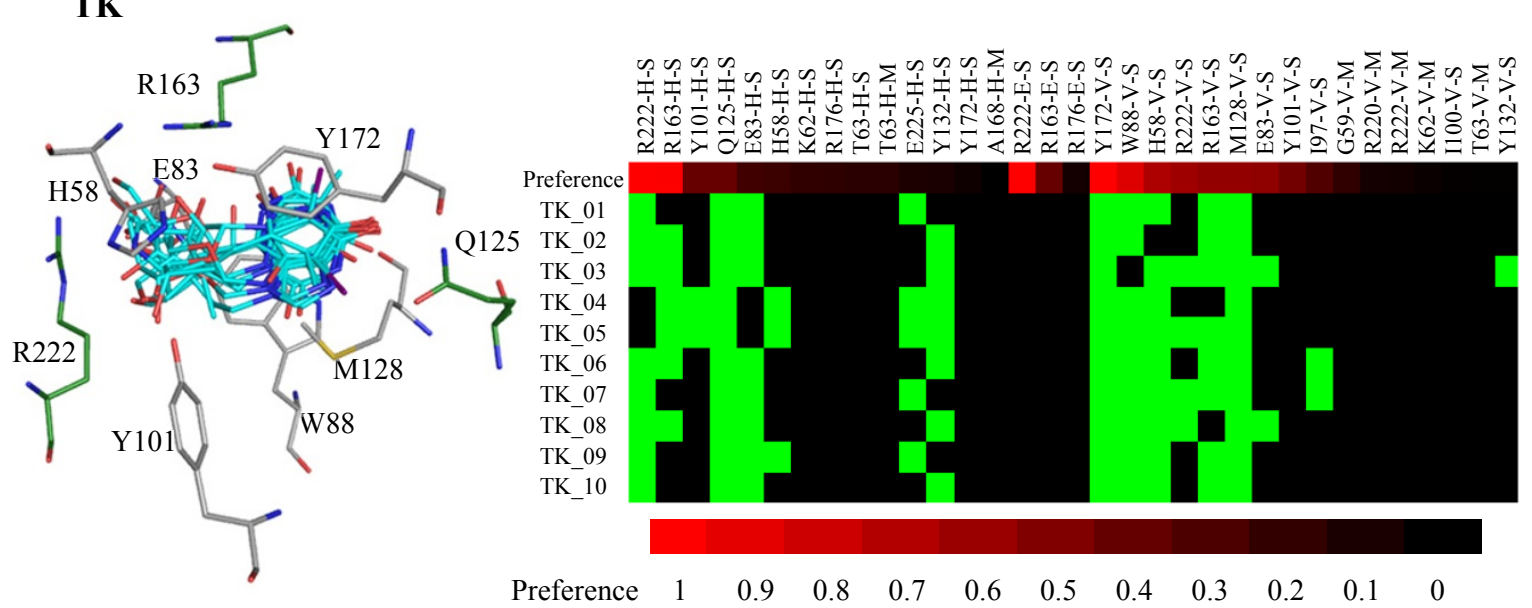

Figure 3 Relationship between the pharmacological interactions and the active compounds of (A) ERA, (B) ER, and (C) TK. The residue with a pharmacological preference $\geq 0.4$ is colored by the interaction types [H: green (E353 and R394 in ERA); E: yellow; and V: gray (L391 and F404 in ERA)]. In the profile, the first row presents the pharmacological preferences of the interacting residue groups using the color-coding bar, with red-through-black indicating high-through-low. The following rows show the interactions between the active compounds and the interacting residue groups. $\mathrm{H}, \mathrm{E}$, and $\mathrm{V}$ indicate the interaction types; $\mathrm{M}$ and $\mathrm{S}$ indicate the main chain and the side chain of the interacting residue, respectively. The hydrogen-bonding or electrostatic interactions are colored in green if the energy $\leq-2.5$. The vdW interactions are colored in green when the energy is less than -4 . 
0.56 , and 0.87 , respectively (Table 1 ). For the substrate binding, M128 and Y172 sandwich the thymine moiety and W88 is a part of the quasi-helical motif [33,34]. These results demonstrated that the pharmacological interactions derived by $i$ GEMDOCK are often involved in the biological functions and the ligand binding.

\section{Molecular docking and virtual screening}

To initially evaluate the utility of $i$ GEMDOCK for docking and virtual screening, we selected a highly diverse dataset with 305 protein-ligand complexes (i.e., CCDC/ Astex set [20] ) and ERA, ER, and TK with 1000 compounds as test sets. Please note that the docking and screening tool of $i$ GEMDOCK is GEMDOCK which was well-studied for VS and some applications [4,8,11-14]. In order to compare with previous works, we followed the docking procedures and performance indices proposed by Nissink, et al. A docked result was considered as a success solution if the root-mean-square derivation (RMSD) $\leq 2.0 \AA$ between the docked solutions and X-ray crystal structures. For these 305 complexes, the success rates of $i$ GEMDOCK and GOLD are 78\% and 68\%, respectively (Table $\mathrm{S} 1$ in additional file 1 ).

The pharmacological scoring function was then applied to identify the active compounds from the 1000 compounds of ERA, ER, and TK. Furthermore, we compared the screening results with those of using the energy-based scoring function of GEMDOCK. These two approaches were tested on the same datasets. The true hits of the three testing sets were used to access the screening accuracy of the two approaches (Fig. S1 in additional file 1). The screening accuracy was generally improved when $i$ GEMDOCK considered the pharmacological interactions.

We compared $i$ GEMDOCK (pharmacological scoring function) with three screening methods (DOCK, GOLD, and FlexX) on the ER and TK sets (Table S2 in additional file 1). To compare with previous works, we followed the experiment design and performance indices used by Bissantz et al. When true-positive rate is $80 \%$, the false positive rates were $2.3 \%$ ( $i$ GEMDOCK), $13.3 \%$ (DOCK), 57.8\% (FlexX), and 5.3\% (GOLD), for ER. The false positive rates were $7.8 \%$ ( $i$ GEMDOCK), $23.4 \%$ (DOCK), 8.8\% (FlexX), and 8.3\% (GOLD) for TK.

\section{Post-screening analysis}

To identify leads from vast amount of docked poses generated during the virtual screening procedure is the key step for the drug discovery. In addition, the topranked compounds based on the scoring functions are not advisable since these compounds may be similar in structures or physico-chemical properties. For these two issues, $i$ GEMDOCK provides a post-screening analysis module to cluster compounds based on the interactions profiles and the atomic compositions. Selecting representative compounds from each cluster is able to maintain compound diversity and then reduces the false positives. Further, when active compounds are available, users can choose the similar compounds in the same cluster of the actives based on hierarchical trees (Fig. 1E). Notably, iGEMDOCK visualizes the interaction profiles of the compounds, and thereby the top-ranked compounds with pharmacological interactions can be selected according to the interaction table (Fig. 2G).

The post-screening analysis module of $i$ GEMDOCK is useful for clustering and selecting compounds based on interaction profiles. We selected a set of compounds, including 10 ERA active compounds and top-ranked 100 compounds based on both the pharmacological and energy-based scoring functions. The hierarchical clustering dendrogram and the profile revealed that the proteinligand interactions derived from the pharmacological scoring function are significantly different from those derived from the energy-based scoring function (Figs. 4A and B). The compounds with the high pharmacological scores and the active compounds consistently keep the pharmacological interactions; conversely, the compounds derived from the energy-based scoring function are often lack of the pharmacological interactions (Fig. 4B). This result indicates the pharmacological interactions are useful for identifying active compounds. For example, the two active compounds, ERA_03 and ERA_06, were ranked as 187 and 160 using the energy-based scoring function, respectively. When the pharmacological interactions were considered, the ranks of ERA_03 and ERA_06 were 91 and 87, respectively (Fig. 4C).

Some compounds having high pharmacological scores are structurally and chemically similar to the active compounds (Fig. 4C). For example, MFCD00003670 (Tetrahydrocortisol) and MFCD00012742 (Tetrahydropapaveroline) were analogues of the ERA active compounds, and both of them were clustered into the same cluster. In addition, the pharmacological scoring function can reduce the ill-effect of most energy-based scoring functions which often favor high molecular weight or highly-polar compounds $[8,35]$. For instance, the ranks of two high molecular weight and polar compounds, MFCD00009772 and MFCD00016940, are 267 and 274, respectively (Fig. 4C). To further examine the pharmacological scoring function, we analyzed the relationship between the molecular weights of the active compounds and the rank improvement using the pharmacological scoring function (Fig. 5). The pharmacological scoring function generally improves the screening accuracy when the molecular weights of the active compounds are less than 400 .

In summary, $i$ GEMDOCK can mine the pharmacological interactions from the screening compounds 
A

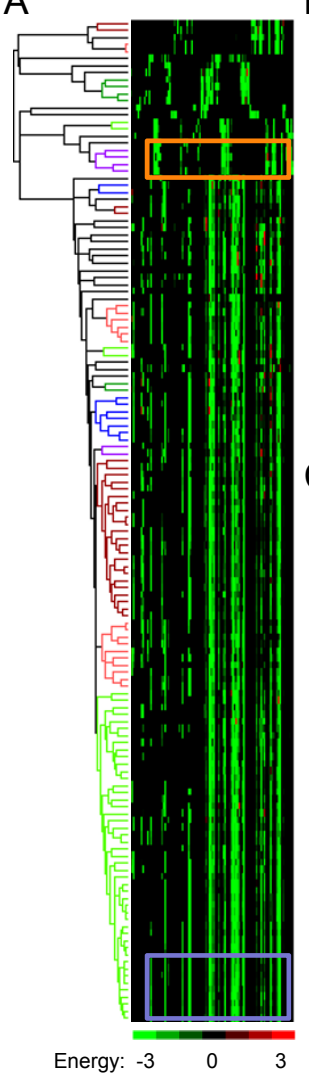

$\mathrm{B}$ 옹

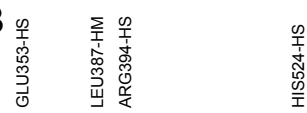

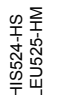

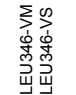

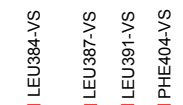
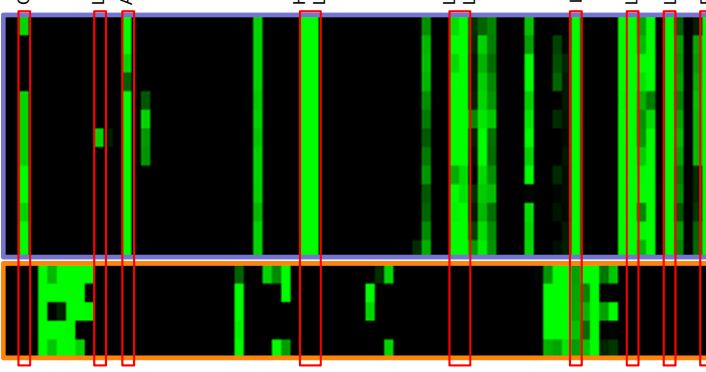

(1)

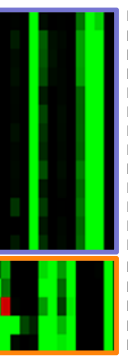

A 05

MFCD00003610 MFCD00003662 ERA_10 ERA-04

ERA_03

ERA-09

MFCD0001274

ERA_06

ERA-01

ERA-02

MFCD00016940

MFCD00013286

MFCD00010058

C
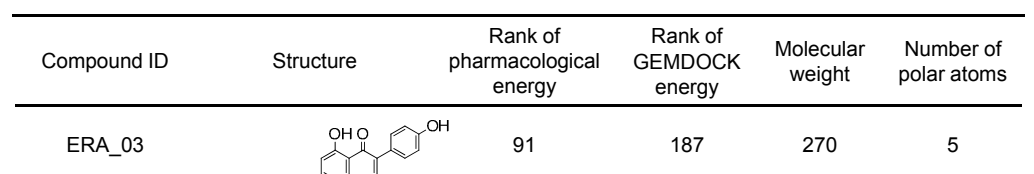

$\mathrm{OH}$

(270

ERA_06

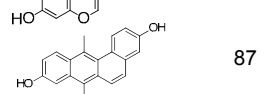

$87 \quad 160$

270

5

MFCD00003670

100

114

288

MFCD00012742

not HO

30

77

367

5

MFCD00009772

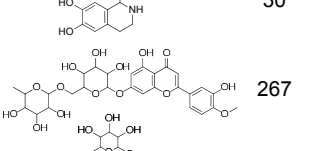

287

5

MFCD00016940

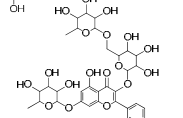

274

609

15

Figure 4 Hierarchical clustering of iGEMDOCK for post screening analysis using ERA as the example. (A) Hierarchical clustering of the interaction profile for the active compounds and top-ranked compounds selected by the pharmacological and energy-based scoring functions.

(B) The interaction profiles of the compounds belonging to the active compound cluster (blue block) and the compounds in the lowest energy cluster (orange block). The red boxes present the pharmacological interactions. (C) The ranks of some typical compounds in the previous two clusters.

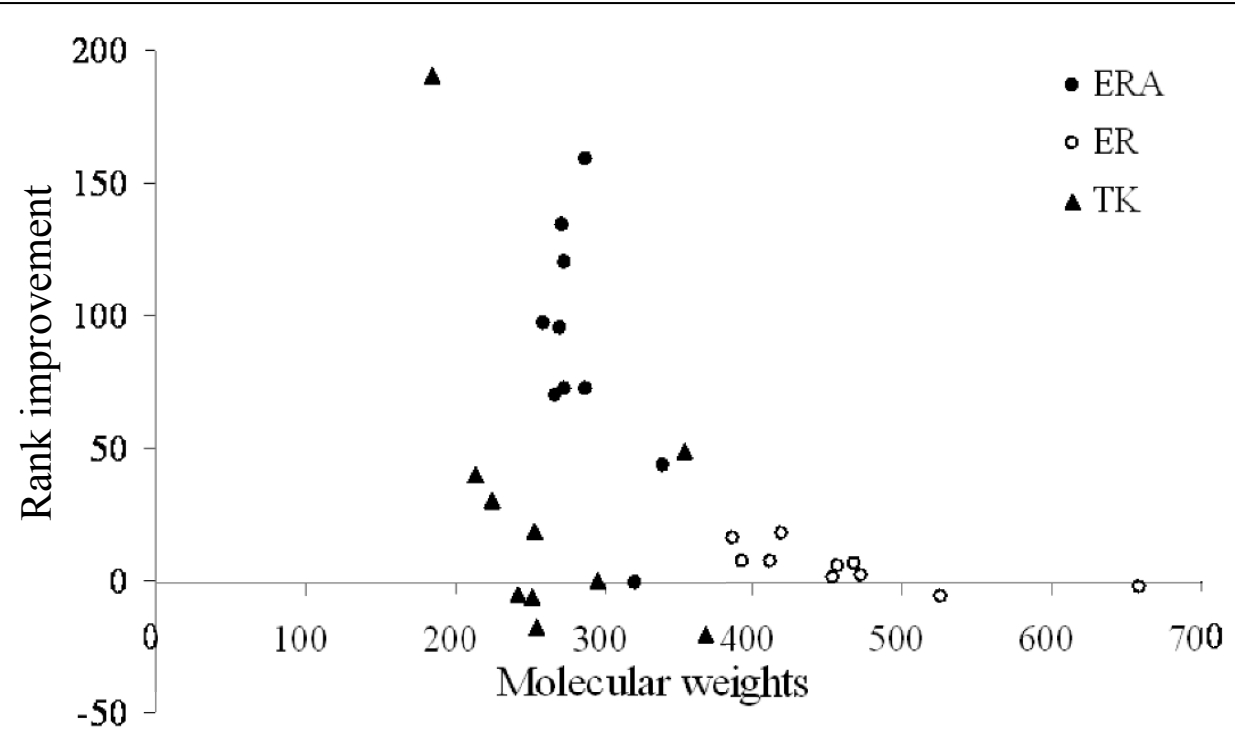

Figure 5 Relationship between the molecular weights of the active compounds and the rank improvement using the pharmacological scoring function for $\operatorname{ERA}(\bullet)$, ER $(0)$, and TK $(\boldsymbol{\bullet})$. 
without known active compounds. Therefore, iGEMDOCK can provide a good starting point for deriving pharmacological interactions (residues) and identifying new potential active compounds for a new protein structure. In addition, $i$ GEMDOCK offers the visualization of the interaction profiles, pharmacological interactions, and the hierarchical clustering dendrogram. Users are able to easily observe and select compounds for post-screening analysis to enrich accuracies.

\section{Conclusions}

We have developed a structure-based VS framework $i$ GEMDOCK from the preparations through to the postscreening analysis. $i$ GEMDOCK is an integrated and easy-to-use environment which is especially useful for post-screening analysis and inferring pharmacological interactions from screening compounds. The friendly user interface is helpful to biologically oriented nonexperts. The experimental results show that the pharmacological interactions are often essential for the binding of the active compounds and involved in biological mechanisms. The pharmacological interactions can reduce the ill effects of energy-based scoring functions to enrich the hit rates in VS. We believe $i$ GEMDOCK is useful for drug discovery and understanding proteinligand mechanisms.

\section{Additional material}

Additional file 1:

\begin{abstract}
Acknowledgements
J-M. Yang was supported by National Science Council and partial support of the ATU plan by MOE. Authors are grateful to both the hardware and the software supports of the Structural Bioinformatics Core Facility at National Chiao Tung University.

This article has been published as part of BMC Bioinformatics Volume 12 Supplement 1, 2011: Selected articles from the Ninth Asia Pacific Bioinformatics Conference (APBC 2011). The full contents of the supplement are available online at http://www.biomedcentral.com/1471-2105/12? issue $=\mathrm{S} 1$.

\section{Author details}

${ }^{1}$ Institute of Bioinformatics and Systems Biology, National Chiao Tung University, Hsinchu, 30050, Taiwan. ${ }^{2}$ Department of Biological Science and Technology, National Chiao Tung University, Hsinchu, 30050, Taiwan. ${ }^{3}$ Core Facility for Structural Bioinformatics, National Chiao Tung University, Hsinchu, 30050, Taiwan
\end{abstract}

\section{Authors' contributions}

$\mathrm{KCH}, \mathrm{YFC}$, and JMY conceived and designed the experiments. YFC, SRL, and JMY implemented the program. KCH, YFC, and JMY performed the experiments and analyzed the data. $\mathrm{KCH}, \mathrm{YFC}$, and JMY wrote the paper.

\section{Competing interests}

The authors declare that they have no competing interests.

Published: 15 February 2011
References

1. Lyne PD: Structure-based virtual screening: an overview. Drug Discovery Today 2002, 7:1047-1055

2. Tanrikulu Y, Schneider G: Pseudoreceptor models in drug design: bridging ligand- and receptor-based virtual screening. Nature Reviews Drug Discovery 2008, 7:667-677.

3. Kitchen DB, Decornez H, Furr JR, Bajorath J: Docking and scoring in virtual screening for drug discovery: methods and applications. Nature Reviews Drug Discovery 2004, 3:935-949.

4. Yang J-M, Chen C-C: GEMDOCK: a generic evolutionary method for molecular docking. Proteins 2004, 55:288-304.

5. Kramer B, Rarey M, Lengauer T: Evaluation of the flexX incremental construction algorithm for protein-ligand docking. Proteins 1999, 37:228-241.

6. Morris GM, Goodsell DS, Huey R, Olson AJ: Distributed automated docking of flexible ligands to proteins: parallel applications of AutoDock 2.4. Journal of Computer-Aided Molecular Design 1996, 10:293-304.

7. Jones $G$, Willett $P$, Glen RC, Leach AR, Taylor R: Development and validation of a genetic algorithm for flexible docking. Journal of Molecular Biology 1997, 267:727-748.

8. Yang JM, Shen TW: A pharmacophore-based evolutionary approach for screening selective estrogen receptor modulators. Proteins 2005, 59(2):205-220.

9. Tafi A, Bernardini C, Botta M, Corelli F, Andreini M, Martinelli A, Ortore G, Baraldi PG, Fruttarolo F, Borea PA, et al: Pharmacophore based receptor modeling: the case of adenosine $\mathrm{A} 3$ receptor antagonists. An approach to the optimization of protein models. Journal of Medicinal Chemistry 2006, 49:4085-4097.

10. Wolber G, Seidel T, Bendix F, Langer T: Molecule-pharmacophore superpositioning and pattern matching in computational drug design. Drug Discovery Today 2008, 13:23-29.

11. Yang JM, Chen YF, Tu YY, Yen KR, Yang YL: Combinatorial computational approaches to identify tetracycline derivatives as flavivirus inhibitors. PLoS One 2007, 2:e428.

12. Chin KH, Lee YC, Tu ZL, Chen $\mathrm{CH}$, Tseng YH, Yang JM, Ryan RP, McCarthy $Y$, Dow JM, Wang AH, et al: The CAMP receptor-like protein CLP is a novel c-di-GMP receptor linking cell-cell signaling to virulence gene expression in Xanthomonas campestris. Journal of Molecular Biology 2010, 396:646-662.

13. Hung HC, Tseng CP, Yang JM, Ju YW, Tseng SN, Chen YF, Chao YS, Hsieh HP, Shih SR, Hsu JT: Aurintricarboxylic acid inhibits influenza virus neuraminidase. Antiviral Research 2009, 81:123-131.

14. Yang M-C, Guan H-H, Yang J-M, Ko C-N, Liu M-Y, Lin Y-H, Chen C-J, Mao SJT: Rational design for crystallization of beta-lactoglobulin and vitamin D-3 complex: revealing a secondary binding site. Crystal Growth \& Design 2008, 8:4268-4276

15. Warnmark A, Treuter E, Gustafsson JA, Hubbard RE, Brzozowski AM, Pike AC: Interaction of transcriptional intermediary factor 2 nuclear receptor box peptides with the coactivator binding site of estrogen receptor alpha. Journal of Biological Chemistry 2002, 277:21862-21868.

16. Shiau AK, Barstad D, Loria PM, Cheng L, Kushner PJ, Agard DA, Greene GL: The structural basis of estrogen receptor/coactivator recognition and the antagonism of this interaction by tamoxifen. Cell 1998, 95:927-937.

17. Champness JN, Bennett MS, Wien F, Visse R, Summers WC, Herdewijn P, de Clerq E, Ostrowski T, Jarvest RL, Sanderson MR: Exploring the active site of herpes simplex virus type- 1 thymidine kinase by X-ray crystallography of complexes with aciclovir and other ligands. Proteins 1998, 32:350-361.

18. Zhou HB, Sheng S, Compton DR, Kim Y, Joachimiak A, Sharma S, Carlson KE, Katzenellenbogen BS, Nettles KW, Greene GL, et al: Structureguided optimization of estrogen receptor binding affinity and antagonist potency of pyrazolopyrimidines with basic side chains. Journal of Medicinal Chemistry 2007, 50:399-403.

19. Manikowski A, Verri A, Lossani A, Gebhardt BM, Gambino J, Focher F, Spadari S, Wright GE: Inhibition of herpes simplex virus thymidine kinases by 2-phenylamino-6-oxopurines and related compounds: structureactivity relationships and antiherpetic activity in vivo. Journal of Medicinal Chemistry 2005, 48:3919-3929.

20. Nissink JW, Murray C, Hartshorn M, Verdonk ML, Cole JC, Taylor R: A new test set for validating predictions of protein-ligand interaction. Proteins 2002, 49:457-471. 
21. Bissantz C, Folkers G, Rognan D: Protein-based virtual screening of chemical databases. 1. Evaluation of different docking/scoring combinations. Journal of Medicinal Chemistry 2000, 43:4759-4767.

22. Sayle RA, Milner-White EJ: RASMOL: biomolecular graphics for all. Trends in Biochemical Sciences 1995, 20:374.

23. Saldanha AJ: Java Treeview-extensible visualization of microarray data. Bioinformatics 2004, 20:3246-3248.

24. Yang J-M, Shen T-W: A pharmacophore-based evolutionary approach for screening selective estrogen receptor modulators. Proteins 2005, 59:205-220.

25. Fradera $X$, Knegtel RM, Mestres J: Similarity-driven flexible ligand docking. Proteins 2000, 40:623-636.

26. Qin Z, Kastrati I, Chandrasena RE, Liu H, Yao P, Petukhov PA, Bolton JL, Thatcher GR: Benzothiophene selective estrogen receptor modulators with modulated oxidative activity and receptor affinity. Journal of Medicinal Chemistry 2007, 50:2682-2692.

27. Manas ES, Xu ZB, Unwalla RJ, Somers WS: Understanding the selectivity of genistein for human estrogen receptor-beta using X-ray crystallography and computational methods. Structure 2004, 12:2197-2207.

28. Brzozowski AM, Pike AC, Dauter Z, Hubbard RE, Bonn T, Engstrom O, Ohman L, Greene GL, Gustafsson JA, Carlquist M: Molecular basis of agonism and antagonism in the oestrogen receptor. Nature 1997 389:753-758.

29. Shadnia H, Wright JS, Anderson JM: Interaction force diagrams: new insight into ligand-receptor binding. Journal of Computer-aided Molecular Design 2009, 23:185-194.

30. Wild K, Bohner T, Folkers G, Schulz GE: The structures of thymidine kinase from herpes simplex virus type 1 in complex with substrates and a substrate analogue. Protein Sci 1997, 6:2097-2106.

31. Kussmann-Gerber S, Kuonen O, Folkers G, Pilger BD, Scapozza L: Drug resistance of herpes simplex virus type 1-structural considerations at the molecular level of the thymidine kinase. European Journal of Biochemistry / FEBS 1998, 255:472-481.

32. Hinds TA, Compadre C, Hurlburt BK, Drake RR: Conservative mutations of glutamine-125 in herpes simplex virus type 1 thymidine kinase result in a ganciclovir kinase with minimal deoxypyrimidine kinase activities. Biochemistry 2000, 39:4105-4111.

33. Pilger BD, Perozzo R, Alber F, Wurth C, Folkers G, Scapozza L: Substrate diversity of herpes simplex virus thymidine kinase. Impact Of the kinematics of the enzyme. The Journal of Biological Chemistry 1999, 274:31967-31973.

34. Evans JS, Lock KP, Levine BA, Champness JN, Sanderson MR, Summers WC McLeish PJ, Buchan A: Herpesviral thymidine kinases: laxity and resistance by design. The Journal of General Virology 1998, 79:2083-2092

35. Pan $Y$, Huang N, Cho S, MacKerell AD Jr.: Consideration of molecular weight during compound selection in virtual target-based database screening. J Chem Inf Comput Sci 2003, 43(1):267-272.

36. Sulpizi M, Schelling P, Folkers G, Carloni P, Scapozza L: The rational of catalytic activity of herpes simplex virus thymidine kinase. a combined biochemical and quantum chemical study. The Journal of Biological Chemistry 2001, 276:21692-21697.

doi:10.1186/1471-2105-12-S1-S33

Cite this article as: Hsu et al:: iGEMDOCK: a graphical environment of enhancing GEMDOCK using pharmacological interactions and postscreening analysis. BMC Bioinformatics 2011 12(Suppl 1):S33.

\section{Submit your next manuscript to BioMed Central and take full advantage of:}

- Convenient online submission

- Thorough peer review

- No space constraints or color figure charges

- Immediate publication on acceptance

- Inclusion in PubMed, CAS, Scopus and Google Scholar

- Research which is freely available for redistribution

Submit your manuscript at www.biomedcentral.com/submit 